\title{
Effect of Integrated Nutrient Management and Plant Geometry on Growth and Quality of Rice (Oryza sativa L.) Varieties under SRI Technique
}

\author{
Harikesh $^{1 *}$, Akhtar Ali ${ }^{1}$, Shivam ${ }^{2}$, Ravi Pratap Yadav ${ }^{1}$, Sanjay Kumar ${ }^{1}$, \\ Ajit Kumar ${ }^{1}$ and Atul Yadav ${ }^{3}$ \\ ${ }^{1}$ Department of Agronomy, ${ }^{2}$ Department of Extension Education, ${ }^{3}$ Department of Horticulture, \\ NDUA\&T, Kumarganj, Faizabad 224229, U.P., India \\ *Corresponding author
}

\section{A B S T R A C T}

The present investigation was conducted with the objective to know the effect of integrated nutrient management and plant geometry on growth and yield attributes of rice varieties during Kharif season of 2015 and 2016. The study comprised six treatments of integrated nutrient management $\left(\mathrm{F}_{0}\right.$ : Control, $\mathrm{F}_{1}: \mathrm{RDF}(120: 60: 60) \quad \mathrm{F}_{2}: 50 \%$ RDF $+50 \%$

\section{Keywords}

Rice varieties, Growth attributes, Quality, SRI.

Article Info

Accepted:

21 September 2017 Available Online: 10 October 2017
Vermicompost, $\mathrm{F}_{3}: 75 \% \mathrm{RDF}+25 \% \mathrm{~N}$ through Vermicompost, $\mathrm{F}_{4}: 50 \% \mathrm{RDF}+50 \% \mathrm{~N}$ through $\mathrm{FYM} \mathrm{F}_{5}: 75 \% \mathrm{RDF}+25 \% \mathrm{~N}$ through FYM) with two varieties namely NDR-359 and Sarju 52 and two plant geometry (a) $\mathrm{S}_{1}: 20 \mathrm{~cm} \times 20 \mathrm{~cm}$. (b) $\mathrm{S}_{2}: 30 \mathrm{~cm} \times 30 \mathrm{~cm}$. the present investigation showed that the growth attributes were significantly influence due to different combination of organic and inorganic nutrients, varieties and plant geometry during both the years and in pooled analysis. The growth attributes viz., plant height $(\mathrm{cm})$, number of shoots $\left(\mathrm{m}^{-2}\right)$, dry matter accumulation $\left(\mathrm{g} \mathrm{m}^{-2}\right)$ at 30, 60, 90 DAT and at harvest and leaf area index at 30,60 and 90 DAT were recorded significantly higher with the application of integrated nutrient management treatment $\mathrm{F}_{2}(50 \% \mathrm{RDF}+50 \% \mathrm{~N}$ through vermicompost). The variety $\mathrm{V}_{1}(\mathrm{NDR}-359)$ and plant geometry $\mathrm{S}_{1}(20 \mathrm{~cm} \times 20 \mathrm{~cm})$ were recorded significantly higher growth attributes viz., plant height $(\mathrm{cm})$, number of shoots $\left(\mathrm{m}^{-2}\right)$, dry matter accumulation and leaf area index under SRI technique during both the year and in pooled analysis. The quality attributes like hulling $\%$ and protein content in grain were recorded in INM treatment $F_{2}$, variety $V_{1}$ and plant geometry $S_{1}$ during both the years and in pooled analysis.

\section{Introduction}

Rice (Oryza sativa L.) is a member of poaceae family and is relished as staple food by majority of world's population. In India, rice occupied 45.16 million hectares area with a production of 103.72 million tonnes and average yield 2.7t ha ${ }^{-1}$ (Anonymous, 2015). Rice is grown in 114 countries across the world on an area about 150 million hectares with annual production of over 525 million tonnes, constituting nearly 11 per cent of the world's cultivated land. The system of Rice Intensification (SRI) was developed by a Jesuit agriculturist Fr. Henri De Laulanie (1993) and Madagascar colleagues working with him in 1980 and the 1990, and they studied ways to increase the low yields of Madagascar farmers. From Madagascar's poor soils which yielded usually an average of 2 tonnes per hectare. SRI system coaxed yield of 6,8 and even 10 tonnes per hectare, while 
reducing the farmers' cost for water, seeds and external inputs. In 1990, Fr. De Laulanie and his colleagues set up an NGO called Association Tefy Saina ('to improve the mind') to develop SRI further and to promote it among Madagascar farmers.

The System of Rice Intensification (SRI) increase opportunity to improve food security through increased rice productivity by changing the management of plants, soil, water and nutrients while reducing external inputs like seed, water, fertilizers and herbicides.

The SRI system has its own methodologies that include transplanting of very young seedling (8-15 days old), transplanting single seedling per hill at square pattern of $25 \mathrm{~cm} \mathrm{x}$ $25 \mathrm{~cm}, 30 \mathrm{~cm} \times 30 \mathrm{~cm}$ (wider spacing), use of mechanical weeder to aerate the soil/plant system, alternate wetting and drying the soil (soil not flooded during the vegetation period) and use of organic manure as nutrient source. Long duration varieties perform better with wider spacing than short duration varieties (Baloch et al., 2002). Stoop (2005) suggested that long-duration varieties perform better under SRI management. Proponents of SRI recommend the use of organic fertilization (compost) instead of chemical fertilizer.

\section{Materials and Methods}

The field experiment was conducted during Kharif 2015 and 2016 at Agronomy Farm of Narendra Deva University of Agriculture and Technology, Narendra Nagar (Kumarganj), Faizabad (U.P.). The experimental site located at Kumarganj is situated $42 \mathrm{~km}$ away from Faizabad city on Faizabad- Raibarely road. Geographically the experimental site is situated at $26.47^{\circ}$ North latitude and $81.12^{\circ}$ East longitude with is an elevation of about $113 \mathrm{~m}$. from mean sea level in the Indo Gangatic Plain Zone of eastern Uttar Pradesh.
The climate in this region is humid and characterized with high rainfall $\left(300 \mathrm{~cm}_{\text {year }}{ }^{-1}\right)$. The soil is sandy to sandy loam with a $\mathrm{pH}$ of 5.05 and $0.72 \%$ organic C. Soil low in available $\mathrm{N}\left(127.92 \mathrm{~kg} \mathrm{ha}^{-1}\right)$, medium in available $\mathrm{P}\left(21.59 \mathrm{~kg} \mathrm{ha}^{-1}\right)$ and low in available $\mathrm{K}\left(122.46 \mathrm{~kg} \mathrm{ha}^{-1}\right)$.

The treatment was carried out with 24 treatment combination formed with six nutrient management levels, two varieties and two levels of plant geometry, in rice which were allocated in split plot design with three replications.

The six irrigation levels (a) $\mathrm{N}_{0}$ : Control (b) $\mathrm{N}_{1}$ : RDF (120:60:60) (c) $\mathrm{N}_{2}: 50 \% \mathrm{RDF}+$ $50 \%$ Vermicompost (d) $\mathrm{N}_{3}: 75 \% \mathrm{RDF}+25 \%$ Vermicompost (e) $\mathrm{N}_{4}: 50 \% \mathrm{RDF}+50 \% \mathrm{FYM}$ (f) $\mathrm{N}_{5}: 75 \% \mathrm{RDF}+25 \% \mathrm{FYM}$ with two varieties namely NDR-359 and Sarju 52 and two plant geometry (a) $S_{1}: 20 \mathrm{~cm} \mathrm{X} 20 \mathrm{~cm}$. (b) $\mathrm{S}_{2}: 30 \mathrm{~cm} \mathrm{X} 30 \mathrm{~cm}$

The crop was fertilised with a uniform dose of $60 \mathrm{~kg} \quad \mathrm{P}_{2} \mathrm{O}_{5} / \mathrm{ha}$ through single super phosphate, $40 \mathrm{~kg} \mathrm{~K}_{2} \mathrm{O} / \mathrm{ha}$ through muriate of potash and half dose of the Nitrogen management as per treatments with organic and inorganic.

The number of shoots were counted at 30,60, 90 DAS and at harvest by placing quadrate at three places in each plot and the plants which come within the quadrate were averaged out to express shoots per square meter Number of effective tillers (ear heads) were counted before harvesting from marked area of one square meter, Total number of spikelets from five selected spikes was counted and average values were recorded. From the individual plot the crop of net plot area was harvested. After air d harvesting and seed were cleaned. The final seed weight was recorded in $\mathrm{kg}$ per plot and converted in to t/ha. 


\section{Results and Discussion}

\section{Effect of integrated nutrient management treatments growth attributes}

Nutrient is the fundamental basis of life. Growth of plant is controlled by rates of cell division, their enlargement and by the supply of organic and inorganic compounds required for the synthesis of new protoplasm and cell wall. Cell enlargement is particularly dependent on least minimum degree of cell turgor. Stem and leaf elongation is quickly checked by nutrient. Thus decreasing nutrient content is accompanied by loss of turgor and wilting, cessation of cell enlargement, closure of stomata, reduction in photosynthesis and interference with many basic metabolic processes.

Integrated Nutrient Management did not change initial plant population significantly because there was uniform nutrient at the time of planting which lead proper standing of crop. This was mainly due to the effect of initial plant population was counted as 5 days of transplanting and nutrient treatments were applied up to this period (Table 1). This might be due to no application of differential irrigation up to this stage (Rath et al., 2010) and (shekara et al., 2011).

Different integrated nutrient management showed significant effect on growth characters viz., plant height and number of shoots $\mathrm{m}^{-2}$ as well as leaf area index at 30, 60, 90 DAT and at harvest stage (Table-2). The plant height show variation significantly due to different nutrient management at growth stage (30 DAT) because variable nutrient was received this stage under different integrated nutrient management, the maximum plant height was recorded with the application of $\mathrm{F}_{2}$ (50\% RDF $+50 \% \mathrm{~N}$ through vermicompost) which, was significantly superior to the $\mathrm{F}_{0}$ (control), $\mathrm{F}_{1}$-RDF (120:60:60), $\mathrm{F}_{5}(75 \% \mathrm{RDF}$ $+25 \% \mathrm{~N}$ through FYM) and $\mathrm{F}_{3}(75 \% \mathrm{RDF}+$
$25 \% \mathrm{~N}$ through vermicompost) at 30, 60, 90 DAT and at harvest. It was statistically at par with treatment $\mathrm{F}_{4}(50 \% \mathrm{RDF}+50 \% \mathrm{~N}$ through FYM). However, the minimum plant height was observed in $\mathrm{F}_{0}$ (control) at 30, 60, 90 DAT and at harvest. Significant reduction in plant height due to decrease in nutrient availability was also reported by Das et al., (2002), Dutt \& Chauhan (2010), Gautam et al., (2012) and Kumar et al.,(2012).

The maximum effect of nutrients level on number of shoots was recorded with the application of $\mathrm{F}_{2}(50 \% \mathrm{RDF}+50 \% \mathrm{~N}$ through vermicompost) which, was significantly higher than $\mathrm{F}_{0}$ (control), $\mathrm{F}_{1}$-RDF (120:60:60), $\mathrm{F}_{5}(75 \% \mathrm{RDF}+25 \% \mathrm{~N}$ through $\mathrm{FYM})$ and $\mathrm{F}_{3}(75 \% \mathrm{RDF}+25 \% \mathrm{~N}$ through vermicompost) (Table-3). It was statistically at par with treatment $\mathrm{F}_{4}(50 \% \mathrm{RDF}+50 \% \mathrm{~N}$ through FYM). However, the minimum number of shoots was observed in $\mathrm{F}_{0}$ (control) at 30, 60, 90 DAT and at harvest.

Similar result was also reported by Rathi and Narine (1990), Das et al., (2002), Mirza et al., (2005), Dutt and Chauhan (2010) and Kumar et al., (2012). In general number of shoots was lowest for initial crop growth period which increased with the increasing crop age. The number of shoots was received maximum during 60-90 DAT and more after it decline gradually (Dutt and Chauhan, 2010).

The dry matter accumulation recorded maximum with the application of $\mathrm{F}_{2}(50 \%$ $\mathrm{RDF}+50 \% \mathrm{~N}$ through vermicompost) which, was significantly higher dry matter accumulation than rest of other INM treatments except treatment $\mathrm{F}_{4}(50 \% \mathrm{RDF}+$ $50 \% \mathrm{~N}$ through FYM). It was statistically at par with treatment $\mathrm{F}_{4}(50 \% \mathrm{RDF}+50 \% \mathrm{~N}$ through FYM). However, the minimum dry matter accumulation was observed in $\mathrm{F}_{0}$ (control) at 30, 60, 90 DAT and at harvest, respectively Vanaja and Raju (2002), Shekhara et al., (2011). 
Table.1 Effect of integrated nutrient management, varieties and plant geometry on initial plant population $\left(\mathrm{m}^{-2}\right)$ of rice under SRI technique

\begin{tabular}{|c|c|c|c|}
\hline \multirow{2}{*}{ Treatment } & \multicolumn{3}{|c|}{ Initial plant population $\left(\mathrm{m}^{-2}\right)$} \\
\hline & 2015 & 2016 & Pooled \\
\hline \multicolumn{4}{|l|}{ Nutrients management } \\
\hline $\mathrm{F}_{0}:$ Control & 18.06 & 18.06 & 18.06 \\
\hline $\mathrm{F}_{1}: \mathrm{RDF}(120: 60: 60)$ & 18.06 & 18.06 & 18.06 \\
\hline $\mathrm{F}_{2}: 50 \% \mathrm{RDF}+50 \% \mathrm{~N}-$ Vermicompost & 18.06 & 18.06 & 18.06 \\
\hline $\mathrm{F}_{3}: 75 \% \mathrm{RDF}+25 \% \mathrm{~N}-$ Vermicompost & 18.06 & 18.06 & 18.06 \\
\hline $\mathrm{F}_{4}: 50 \% \mathrm{RDF}+50 \% \mathrm{~N}-\mathrm{FYM}$ & 18.06 & 18.06 & 18.06 \\
\hline $\mathrm{F}_{5}: 75 \% \mathrm{RDF}+25 \% \mathrm{~N}-\mathrm{FYM}$ & 18.06 & 18.06 & 18.06 \\
\hline $\mathrm{SEm} \pm$ & 0.34 & 0.34 & 0.24 \\
\hline $\mathrm{CD}$ at $5 \%$ & NS & NS & NS \\
\hline \multicolumn{4}{|l|}{ Varieties } \\
\hline $\mathrm{V}_{1}:$ NDR-359 & 18.06 & 18.06 & 18.06 \\
\hline $\mathrm{V}_{2}:$ Sarju 52 & 18.06 & 18.06 & 18.06 \\
\hline $\mathrm{SEm} \pm$ & 0.20 & 0.20 & 0.14 \\
\hline $\mathrm{CD}$ at $5 \%$ & NS & NS & NS \\
\hline \multicolumn{4}{|l|}{ Plant geometry } \\
\hline $\mathrm{S}_{1}: 20 \mathrm{~cm} \times 20 \mathrm{~cm}$ & 25.00 & 25.00 & 25.00 \\
\hline $\mathrm{S}_{2}: 30 \mathrm{~cm} \times 30 \mathrm{~cm}$ & 11.11 & 11.11 & 11.11 \\
\hline $\mathrm{SEm} \pm$ & 0.20 & 0.20 & 0.14 \\
\hline $\mathrm{CD}$ at $5 \%$ & 0.57 & 0.57 & 0.40 \\
\hline
\end{tabular}


Table.2 Effect of integrated nutrient management, varieties and plant geometry on plant height (cm) of rice under SRI technique

\begin{tabular}{|c|c|c|c|c|c|c|c|c|c|c|c|c|}
\hline \multirow{2}{*}{ Treatment } & \multicolumn{3}{|c|}{ Plant height $(\mathrm{cm})$ at 30 DAT } & \multicolumn{3}{|c|}{ Plant height $(\mathrm{cm})$ at 60 DAT } & \multicolumn{3}{|c|}{ Plant height $(\mathrm{cm})$ at 90 DAT } & \multicolumn{3}{|c|}{ Plant height $(\mathrm{cm})$ at harvest } \\
\hline & 2015 & 2016 & Pooled & 2015 & 2016 & Pooled & 2015 & 2016 & Pooled & 2015 & 2016 & Pooled \\
\hline \multicolumn{13}{|c|}{ Nutrients management } \\
\hline $\mathrm{F}_{0}$ & 36.19 & 37.15 & 36.67 & 60.06 & 61.75 & 60.91 & 74.07 & 75.31 & 74.69 & 76.62 & 77.92 & 77.27 \\
\hline $\mathrm{F}_{1}$ & 47.47 & 48.73 & 48.10 & 78.78 & 81.00 & 79.89 & 97.16 & 98.78 & 97.97 & 100.50 & 102.21 & 101.35 \\
\hline $\mathrm{F}_{2}$ & 51.70 & 53.08 & 52.39 & 85.80 & 88.22 & 87.01 & 105.82 & 107.58 & 106.70 & 109.45 & 111.32 & 110.39 \\
\hline $\mathrm{F}_{3}$ & 48.88 & 50.18 & 49.53 & 81.12 & 83.41 & 82.26 & 100.05 & 101.71 & 100.88 & 103.48 & 105.25 & 104.36 \\
\hline $\mathrm{F}_{4}$ & 49.82 & 51.15 & 50.48 & 82.68 & 85.01 & 83.85 & 101.97 & 103.67 & 102.82 & 105.47 & 107.27 & 106.37 \\
\hline $\mathrm{F}_{5}$ & 47.94 & 49.22 & 48.58 & 79.56 & 81.80 & 80.68 & 98.12 & 99.76 & 98.94 & 101.49 & 103.22 & 102.36 \\
\hline $\mathrm{SEm} \pm$ & 0.90 & 0.92 & 0.64 & 1.48 & 1.53 & 1.06 & 1.83 & 1.89 & 1.32 & 1.89 & 1.96 & 1.36 \\
\hline $\mathrm{CD}$ at $5 \%$ & 2.57 & 2.61 & 1.92 & 4.22 & 4.34 & 2.99 & 5.21 & 5.38 & 3.91 & 5.39 & 5.57 & 4.05 \\
\hline \multicolumn{13}{|l|}{ Varieties } \\
\hline $\mathrm{V}_{1}$ & 47.94 & 49.22 & 48.58 & 80.89 & 83.17 & 82.03 & 99.76 & 101.42 & 100.59 & 103.18 & 104.94 & 104.06 \\
\hline $\mathrm{V}_{2}$ & 46.06 & 47.29 & 46.67 & 75.11 & 77.23 & 76.17 & 92.64 & 94.18 & 93.41 & 95.82 & 97.46 & 96.64 \\
\hline SEm \pm & 0.52 & 0.53 & 0.37 & 0.86 & 0.88 & 0.61 & 1.06 & 1.09 & 0.76 & 1.09 & 1.13 & 0.79 \\
\hline $\mathrm{CD}$ at $5 \%$ & 1.48 & 1.51 & 1.04 & 2.44 & 2.51 & 3.17 & 3.01 & 3.11 & 2.13 & 3.11 & 3.22 & 2.21 \\
\hline \multicolumn{13}{|c|}{ Plant geometry } \\
\hline$\overline{S_{1}}$ & 48.41 & 49.70 & 49.05 & 80.34 & 82.61 & 81.47 & 99.09 & 100.73 & 99.91 & 102.49 & 104.24 & 103.36 \\
\hline $\mathrm{S}_{2}$ & 45.59 & 46.80 & 46.20 & 75.66 & 77.79 & 76.73 & 93.31 & 94.87 & 94.09 & 96.52 & 98.16 & 97.34 \\
\hline SEm \pm & 0.52 & 0.53 & 0.37 & 0.86 & 0.88 & 0.61 & 1.06 & 1.09 & 0.76 & 1.09 & 1.13 & 0.79 \\
\hline $\mathrm{CD}$ at $5 \%$ & 1.48 & 1.51 & 1.04 & 2.44 & 2.51 & 1.73 & 3.01 & 3.11 & 2.13 & 3.11 & 3.22 & 2.21 \\
\hline
\end{tabular}


Table.3 Effect of integrated nutrient management, varieties and plant geometry on number of shoots $\left(\mathrm{m}^{-2}\right)$ of rice under SRI technique

\begin{tabular}{|c|c|c|c|c|c|c|c|c|c|c|c|c|}
\hline \multirow[t]{2}{*}{ Treatment } & \multicolumn{3}{|c|}{$\begin{array}{c}\text { Number of shoots }\left(\mathrm{m}^{-2}\right) \text { at } 30 \\
\text { DAT }\end{array}$} & \multicolumn{3}{|c|}{$\begin{array}{c}\text { Number of shoots }\left(\mathrm{m}^{-2}\right) \text { at } 60 \\
\text { DAT }\end{array}$} & \multicolumn{3}{|c|}{$\begin{array}{c}\text { Number of shoots }\left(\mathrm{m}^{-2}\right) \text { at } 90 \\
\text { DAT }\end{array}$} & \multicolumn{3}{|c|}{$\begin{array}{c}\text { Number of shoots }\left(\mathrm{m}^{-2}\right) \text { at } \\
\text { harvest }\end{array}$} \\
\hline & 2015 & 2016 & Pooled & 2015 & 2016 & Pooled & 2015 & 2016 & Pooled & 2015 & 2016 & Pooled \\
\hline \multicolumn{13}{|c|}{ Nutrients management } \\
\hline $\mathrm{F}_{0}$ & 126.92 & 130.22 & 128.57 & 270.06 & 279.70 & 274.88 & 270.90 & 281.85 & 276.38 & 245.88 & 250.80 & 248.34 \\
\hline $\mathrm{F}_{1}$ & 187.04 & 191.85 & 189.44 & 397.88 & 412.09 & 404.98 & 444.80 & 462.78 & 453.79 & 403.72 & 411.79 & 407.75 \\
\hline $\mathrm{F}_{2}$ & 194.01 & 199.00 & 196.50 & 412.70 & 427.44 & 420.07 & 558.40 & 580.97 & 569.69 & 506.83 & 516.96 & 511.89 \\
\hline $\mathrm{F}_{3}$ & 190.24 & 195.13 & 192.69 & 404.69 & 419.14 & 411.91 & 504.00 & 524.37 & 514.19 & 457.45 & 466.59 & 462.02 \\
\hline $\mathrm{F}_{4}$ & 192.13 & 197.06 & 194.60 & 408.69 & 423.29 & 415.99 & 531.20 & 552.67 & 541.94 & 482.14 & 491.78 & 486.96 \\
\hline $\mathrm{F}_{5}$ & 189.68 & 194.55 & 192.12 & 403.48 & 417.89 & 410.69 & 481.95 & 501.43 & 491.69 & 437.43 & 446.18 & 441.81 \\
\hline $\mathrm{SEm} \pm$ & 3.42 & 3.49 & 2.44 & 7.28 & 7.51 & 5.23 & 9.24 & 9.62 & 6.67 & 8.39 & 8.56 & 5.99 \\
\hline $\mathrm{CD}$ at $5 \%$ & 9.73 & 9.95 & 6.87 & 20.74 & 21.37 & 14.69 & 26.31 & 27.37 & 18.73 & 23.88 & 24.35 & 16.83 \\
\hline \multicolumn{13}{|l|}{ Varieties } \\
\hline $\mathrm{V}_{1}$ & 183.43 & 191.77 & 187.60 & 397.71 & 411.92 & 404.81 & 473.60 & 492.74 & 483.17 & 429.86 & 438.45 & 434.15 \\
\hline $\mathrm{V}_{2}$ & 176.57 & 177.50 & 177.04 & 368.12 & 381.27 & 374.70 & 456.82 & 475.28 & 466.05 & 414.62 & 422.91 & 418.77 \\
\hline SEm \pm & 1.97 & 2.02 & 1.41 & 4.21 & 4.33 & 3.02 & 5.34 & 5.55 & 3.85 & 4.84 & 4.94 & 3.46 \\
\hline $\mathrm{CD}$ at $5 \%$ & 5.62 & 5.74 & 3.96 & 11.97 & 12.34 & 8.48 & 15.19 & 15.80 & 10.81 & 13.79 & 14.06 & 9.71 \\
\hline \multicolumn{13}{|c|}{ Plant geometry } \\
\hline $\mathrm{S}_{1}$ & 187.02 & 191.84 & 189.43 & 397.85 & 412.06 & 404.96 & 506.83 & 527.32 & 517.08 & 460.02 & 469.22 & 464.62 \\
\hline $\mathrm{S}_{2}$ & 172.98 & 177.43 & 175.21 & 367.98 & 381.12 & 374.55 & 423.58 & 440.70 & 432.14 & 384.46 & 392.14 & 388.30 \\
\hline SEm \pm & 1.97 & 2.02 & 1.41 & 4.21 & 4.33 & 3.02 & 5.34 & 5.55 & 3.85 & 4.84 & 4.94 & 3.46 \\
\hline $\mathrm{CD}$ at $5 \%$ & 5.62 & 5.74 & 3.96 & 11.97 & 12.34 & 8.48 & 15.19 & 15.80 & 10.81 & 13.79 & 14.06 & 9.71 \\
\hline
\end{tabular}


Table.4 Effect of integrated nutrient management, varieties and plant geometry on dry matter accumulation $\left(\mathrm{g} \mathrm{m}^{-2}\right)$ of rice under SRI technique

\begin{tabular}{|c|c|c|c|c|c|c|c|c|c|c|c|c|}
\hline \multirow[t]{2}{*}{ Treatment } & \multicolumn{3}{|c|}{$\begin{array}{l}\text { Dry matter accumulation at } \\
\text { 30 DAT }\end{array}$} & \multicolumn{3}{|c|}{$\begin{array}{l}\text { Dry matter accumulation at } \\
\text { 60DAT }\end{array}$} & \multicolumn{3}{|c|}{$\begin{array}{l}\text { Dry matter accumulation at } \\
\text { 90DAT }\end{array}$} & \multicolumn{3}{|c|}{$\begin{array}{c}\text { Dry matter accumulation at } \\
\text { harvest }\end{array}$} \\
\hline & 2015 & 2016 & Pooled & 2015 & 2016 & Pooled & 2015 & 2016 & Pooled & 2015 & 2016 & Pooled \\
\hline \multicolumn{13}{|c|}{ Nutrients management } \\
\hline $\mathrm{F}_{0}$ & 98.24 & 108.24 & 103.24 & 274.54 & 320.31 & 297.42 & 439.07 & 447.54 & 443.30 & 627.90 & 632.30 & 630.10 \\
\hline $\mathrm{F}_{1}$ & 137.30 & 146.96 & 142.13 & 545.80 & 574.33 & 560.07 & 720.92 & 734.82 & 727.87 & 1030.96 & 1038.19 & 1034.58 \\
\hline $\mathrm{F}_{2}$ & 152.84 & 163.59 & 158.22 & 585.28 & 615.89 & 600.59 & 905.04 & 922.49 & 913.77 & 1294.27 & 1303.34 & 1298.80 \\
\hline $\mathrm{F}_{3}$ & 146.60 & 158.57 & 152.59 & 572.17 & 602.09 & 587.13 & 816.87 & 832.62 & 924.25 & 1168.18 & 1176.37 & 1172.27 \\
\hline $\mathrm{F}_{4}$ & 147.30 & 159.35 & 153.32 & 575.18 & 605.29 & 590.24 & 860.96 & 877.56 & 859.26 & 1231.22 & 1239.85 & 1235.54 \\
\hline $\mathrm{F}_{5}$ & 137.25 & 148.73 & 142.99 & 550.04 & 578.75 & 564.40 & 781.13 & 796.19 & 788.66 & 1117.06 & 1124.89 & 1120.98 \\
\hline SEm \pm & 2.60 & 2.79 & 1.91 & 9.82 & 10.42 & 7.16 & 14.98 & 15.27 & 10.69 & 21.42 & 21.57 & 15.20 \\
\hline CD at $5 \%$ & 7.39 & 7.95 & 5.66 & 27.97 & 29.67 & 20.11 & 42.64 & 43.86 & 30.06 & 60.98 & 61.40 & 42.69 \\
\hline \multicolumn{13}{|l|}{ Varieties } \\
\hline $\mathrm{V}_{1}$ & 144.66 & 156.07 & 150.37 & 535.30 & 569.53 & 552.41 & 767.60 & 782.40 & 775.00 & 1097.72 & 1105.41 & 1101.56 \\
\hline $\mathrm{V}_{2}$ & 128.35 & 138.91 & 133.63 & 499.03 & 529.37 & 514.20 & 740.40 & 754.67 & 747.54 & 1058.81 & 1066.24 & 1062.53 \\
\hline SEm \pm & 1.50 & 1.61 & 1.10 & 5.67 & 6.02 & 4.13 & 8.65 & 8.81 & 6.17 & 12.37 & 12.45 & 8.78 \\
\hline CD at $5 \%$ & 4.27 & 4.59 & 3.09 & 16.15 & 17.13 & 11.61 & 24.62 & 25.09 & 17.34 & 35.20 & 35.45 & 24.65 \\
\hline \multicolumn{13}{|c|}{ Plant geometry } \\
\hline $\mathrm{S}_{1}$ & 143.54 & 154.42 & 148.98 & 535.60 & 568.98 & 552.29 & 821.47 & 737.31 & 729.39 & 1174.75 & 1182.98 & 1178.86 \\
\hline $\mathrm{S}_{2}$ & 129.47 & 140.55 & 135.01 & 498.74 & 529.92 & 514.33 & 686.53 & 699.77 & 693.15 & 981.78 & 988.67 & 985.23 \\
\hline SEm \pm & 1.50 & 1.61 & 1.10 & 5.67 & 6.02 & 4.13 & 8.65 & 8.81 & 6.17 & 12.37 & 12.45 & 8.78 \\
\hline CD at $5 \%$ & 4.27 & 4.59 & 3.09 & 16.15 & 17.13 & 11.61 & 24.62 & 25.09 & 17.34 & 35.20 & 35.45 & 24.65 \\
\hline
\end{tabular}


Table.5 Effect of integrated nutrient management, varieties and plant geometry on leaf area index of rice under SRI technique

\begin{tabular}{|c|c|c|c|c|c|c|c|c|c|}
\hline \multirow{2}{*}{ Treatment } & \multicolumn{3}{|c|}{ Leaf area index at $30 \mathrm{DAT}$} & \multicolumn{3}{|c|}{ Leaf area index at $60 \mathrm{DAT}$} & \multicolumn{3}{|c|}{ Leaf area index at $90 \mathrm{DAT}$} \\
\hline & 2015 & 2016 & Pooled & 2015 & 2016 & Pooled & 2015 & 2016 & Pooled \\
\hline \multicolumn{10}{|c|}{ Nutrients management } \\
\hline $\mathrm{F}_{0}$ & 2.50 & 2.57 & 2.53 & 3.45 & 3.49 & 3.47 & 3.75 & 3.81 & 3.78 \\
\hline $\mathrm{F}_{1}$ & 2.78 & 2.86 & 2.82 & 3.79 & 3.83 & 3.81 & 4.12 & 4.19 & 4.16 \\
\hline $\mathrm{F}_{2}$ & 2.88 & 2.97 & 2.93 & 3.93 & 3.98 & 3.96 & 4.27 & 4.35 & 4.31 \\
\hline $\mathrm{F}_{3}$ & 2.83 & 2.91 & 2.87 & 3.86 & 3.90 & 3.88 & 4.19 & 4.26 & 4.23 \\
\hline $\mathrm{F}_{4}$ & 2.86 & 2.94 & 2.90 & 3.90 & 3.94 & 3.92 & 4.23 & 4.30 & 4.27 \\
\hline $\mathrm{F}_{5}$ & 2.82 & 2.90 & 2.86 & 3.85 & 3.89 & 3.87 & 4.18 & 4.25 & 4.21 \\
\hline $\mathrm{SEm} \pm$ & 0.05 & 0.05 & 0.04 & 0.07 & 0.07 & 0.05 & 0.08 & 0.08 & 0.06 \\
\hline $\mathrm{CD}$ at $5 \%$ & 0.15 & 0.16 & 0.11 & 0.21 & 0.21 & 0.15 & 0.23 & 0.23 & 0.16 \\
\hline \multicolumn{10}{|l|}{ Varieties } \\
\hline $\mathrm{V}_{1}$ & 2.90 & 2.98 & 2.94 & 3.96 & 4.00 & 3.98 & 4.30 & 4.38 & 4.34 \\
\hline $\mathrm{V}_{2}$ & 2.66 & 2.73 & 2.70 & 3.63 & 3.67 & 3.65 & 3.95 & 4.01 & 3.98 \\
\hline SEm \pm & 0.03 & 0.03 & 0.02 & 0.04 & 0.04 & 0.03 & 0.05 & 0.05 & 0.03 \\
\hline $\mathrm{CD}$ at $5 \%$ & 0.09 & 0.09 & 0.06 & 0.12 & 0.12 & 0.08 & 0.13 & 0.13 & 0.09 \\
\hline \multicolumn{10}{|l|}{ Plant geometry } \\
\hline $\mathrm{S}_{1}$ & 2.86 & 2.95 & 2.91 & 3.91 & 3.95 & 3.93 & 4.25 & 4.32 & 4.28 \\
\hline $\mathrm{S}_{2}$ & 2.69 & 2.77 & 2.73 & 3.68 & 3.72 & 3.70 & 4.00 & 4.07 & 4.03 \\
\hline SEm \pm & 0.03 & 0.03 & 0.02 & 0.04 & 0.04 & 0.03 & 0.05 & 0.05 & 0.03 \\
\hline $\mathrm{CD}$ at $5 \%$ & 0.09 & 0.09 & 0.06 & 0.12 & 0.12 & 0.08 & 0.13 & 0.13 & 0.09 \\
\hline
\end{tabular}


Table.6 Effect of integrated nutrient management, varieties and plant geometry on Hulling, protein content in grain and $\mathrm{N}$ uptake of rice under SRI technique

\begin{tabular}{|c|c|c|c|c|c|c|c|c|c|}
\hline \multirow{2}{*}{ Treatment } & \multicolumn{3}{|c|}{ Hulling (\%) } & \multicolumn{3}{|c|}{ Protein content in grain $(\%)$} & \multicolumn{3}{|c|}{ Total $N$ uptake $\left(\mathrm{kg} \mathrm{ha}^{-1}\right)$} \\
\hline & 2015 & 2016 & Pooled & 2015 & 2016 & Pooled & 2015 & 2016 & Pooled \\
\hline \multicolumn{10}{|c|}{ Nutrients management } \\
\hline $\mathrm{F}_{0}$ & 64.76 & 66.06 & 65.41 & 7.02 & 7.09 & 7.06 & 59.61 & 63.16 & 61.38 \\
\hline $\mathrm{F}_{1}$ & 65.43 & 66.08 & 65.75 & 7.15 & 7.22 & 7.18 & 78.78 & 83.58 & 81.18 \\
\hline $\mathrm{F}_{2}$ & 68.21 & 68.89 & 68.55 & 7.84 & 7.91 & 7.88 & 93.53 & 99.05 & 96.29 \\
\hline $\mathrm{F}_{3}$ & 67.02 & 67.55 & 67.29 & 7.40 & 7.47 & 7.44 & 84.62 & 89.77 & 87.19 \\
\hline $\mathrm{F}_{4}$ & 67.70 & 68.38 & 68.04 & 7.68 & 7.76 & 7.72 & 89.02 & 94.38 & 91.70 \\
\hline $\mathrm{F}_{5}$ & 66.11 & 66.78 & 66.44 & 7.27 & 7.35 & 7.31 & 80.91 & 85.85 & 83.38 \\
\hline SEm \pm & 1.30 & 1.31 & 0.92 & 0.14 & 0.15 & 0.10 & 1.55 & 1.65 & 1.13 \\
\hline $\mathrm{CD}$ at $5 \%$ & 3.69 & 3.74 & 2.59 & 0.41 & 0.41 & 0.29 & 4.42 & 4.72 & 3.18 \\
\hline \multicolumn{10}{|l|}{ Varieties } \\
\hline $\mathrm{V}_{1}$ & 66.86 & 67.74 & 67.30 & 7.40 & 7.48 & 7.44 & 84.23 & 89.29 & 86.76 \\
\hline $\mathrm{V}_{2}$ & 66.22 & 66.84 & 66.53 & 7.39 & 7.46 & 7.42 & 77.92 & 82.64 & 80.28 \\
\hline SEm \pm & 0.75 & 0.76 & 0.53 & 0.08 & 0.08 & 0.06 & 0.90 & 0.95 & 0.65 \\
\hline $\mathrm{CD}$ at $5 \%$ & 2.13 & 2.16 & 1.50 & NS & NS & NS & 2.55 & 2.71 & 1.84 \\
\hline \multicolumn{10}{|l|}{ Plant geometry } \\
\hline $\mathrm{S}_{1}$ & 66.75 & 67.37 & 67.06 & 7.36 & 7.43 & 7.39 & 83.04 & 88.04 & 85.54 \\
\hline$\overline{S_{2}}$ & 66.33 & 67.21 & 66.77 & 7.43 & 7.50 & 7.47 & 79.12 & 83.89 & 81.51 \\
\hline SEm \pm & 0.75 & 0.76 & $\mathbf{0 . 5 3}$ & 0.08 & 0.08 & 0.06 & 0.90 & 0.95 & 0.65 \\
\hline $\mathrm{CD}$ at $5 \%$ & 2.13 & 2.16 & 1.50 & NS & NS & NS & 2.55 & 2.71 & 1.84 \\
\hline
\end{tabular}


The leaf area index was increased very slowly over a fairly long period (60 DAS) and ushered in a period of rapid expansion possibly because of increased light absorption and high photosynthetic activities. As leaf area increases, light absorption and rate of dry matter production increase till the foliage become sufficiently dense to cause mutual shading resulting reduced photosynthetic activity of lower leaves.

Leaf area index was also significantly influenced by nutrient management at 30,60 and 90 DAT. Significantly higher leaf area index was observed with the application of $\mathrm{F}_{2}$ (50\% RDF $+50 \% \mathrm{~N}$ through vermicompost) which was significantly higher than control $\left(\mathrm{F}_{0}\right)$. It was statistically at par with $\mathrm{F}_{1}-\mathrm{RDF}$ $(120: 60: 60), \mathrm{F}_{3}(75 \% \mathrm{RDF}+25 \% \mathrm{~N}-$ through Vermicompost), $\mathrm{F}_{4}(50 \% \mathrm{RDF}+50 \%$ $\mathrm{N}$ - through FYM) and $\mathrm{F}_{5}(75 \% \mathrm{RDF}+25 \% \mathrm{~N}-$ through FYM).

Increase in leaf area index with increasing nutrient availability might be due to the fact that nutrient supply contributed towards to more number of green leaves, which attributed to high leaf area and leaf area index. The lowest leaf area index was recorded under in $\mathrm{F}_{0}$ (control). The results were in proximity to those of Sharma and Mitra (1992) and (Shankar and Laware, 2011).

\section{Effect of varieties on growth attributes}

Plant growth and yield are governed by the combined effects of inherited genetic potential and environment in which plants are grown. There is vital role of selection of cultivar in paddy crop because of the fact that variation in the duration, photo-sensitiveness, thermo-sensitiveness and vegetative lag period of the variety. A variety of short duration completes their life cycle with in short period with less effect of photoperiod and low temperature. Longer duration varieties and photo and thermo insensitive varieties may perform better under favorable conditions.

The significant difference was not found in the initial plant population among the varieties. While significant differences in plant height, number of shoot $\mathrm{m}^{-2}$, LAI and dry matter accumulation were recorded with variety NDR-359 at all the growth stage of crops. Similarly another growth characteristics and yield attributing characters showed the significant differences among the varieties. The variation in growth development and yield of varieties might be due to their genetic characteristics. Similar finding in respect to varieties were also reported by Dey et al., (2006), Islam et al., (2008), Limochi et al., (2012) and Tyeb et al., (2013).

Harvest index is the function of grain and straw yield which was significantly equal in both the varieties.

Among the cultivars of wheat included in experiment, NDR-359 has been found more promising in comparison to Sarju-52 to provide higher values of nutrient uptake as well as quality (protein content) during both of years.

The plant height recorded at all the stages of observations of crop was significantly higher in rice cultivar NDR-359 than the Sarjy-52 cultivars. The taller plant in cultivar NDR-359 might be due to better utilization of available growth resources like light and temperature which may result in more nitrogen absorption for the synthesis of protoplasm responsible for rapid cell division consequently increasing the plant in shape and size or may be due to vigour of the cultivar. Similar findings have also been reported by Dey et al., (2006) Singh and Singh (2008) and Awasthe et al., (2011). 
The higher number of total tillers $\mathrm{m}^{-2}$ were recorded in cultivar NDR-359 (Table 4) might be due to ability of effective utilization of plant growth resources viz. photoperiod, dry matter production and increase in tillers with advancement of life cycle. The findings have also been supported by Singh and Singh (2008) and Dey et al., (2006). The dry matter accumulation $\mathrm{m}^{-1}$ running row recorded at all the stages of observations of crop was significantly higher in cultivar NDR-359 than sarju-52 (Table 4). The higher dry matter accumulation $\mathrm{m}^{-1}$ running row in cultivar NDR-359 might be due significantly higher dry matter production $\mathrm{m}^{-1}$ running row at all the stages of observations.

The rice cultivar NDR-359 recorded significantly higher LAI at all the stages of observations than the rest of the Sarju-52 cultivar (Table 5). The higher LAI recorded in cultivar NDR-359 might be due to more number of leaves $\mathrm{m}^{-2}$. The findings have also been supported by Singh and Singh (2008). At 90 DAT leaf area index of NDR-359 was not increased as high as other cultivars as it was a short duration crop and number of leaves $\mathrm{m}^{-2}$ were decreased at 90 DAS but leaf size increased. Thus leaf area index slightly increased reported by Dey et al., (2006).

\section{Quality traits}

\section{Hulling (\%)}

The hulling (\%) was not influenced due to INM treatments, varieties and plant geometry. This result similarly found by Subhash et al., (2005).

\section{Protein content}

The protein content in grain significantly influenced with application of various organic manures and fertilizers. The maximum protein content in grain was found with the application $\mathrm{F}_{2}(50 \% \mathrm{RDF}+50 \% \mathrm{~N}$ through vermicompost) which was significantly higher over the $F_{0}, F_{1}, F_{3}$ and $F_{5}$ INM treatments but at par with the $\mathrm{F}_{4}$ INM treatment The lowest protein content was recorded in the treatment received only $\mathrm{F}_{0}$ (Table-6). This was due to high nitrogen content and uptake by grains receiving sufficient and balanced nutrition. This might be attributed to better root development and higher nitrogen utilization by crop under adequate supply of $\mathrm{N}, \mathrm{P}, \mathrm{K}$ which enhanced the protein synthesis and ultimately increased protein content in grains. Dixit and Gupta (2000) reported that application of FYM @ $10 \mathrm{t} \mathrm{ha}^{-1}$ combined with BGA inoculation increased the protein and amylase content in rice. Varieties did not have significant effect on protein content and the plant geometry also $S_{1}(20 \mathrm{~cm} \times 20 \mathrm{~cm})$ and $S_{2}(30 \mathrm{~cm} \times 30 \mathrm{~cm})$ did not have significant effect on protein content (Singh and Verma, 2006; Dewedi et al., 2006).

\section{References}

Anonymous, 2015 Economics survey of India, Economic Division Ministry of Finance, Government, of India, New Delhi pp 45-55.

Awasthe, R.K., Verma, S., Kumar, A. and Rahaman, H. 2011. Performance of rice variety at different spacing under SRI of in mid. hill add soil of Sikkim Himalayas. Indian Journal of Agronomy, 57 (1):32-37.

Baloch, A. W., Soomro, A. M., Javed, M. A., Ahmed, M., Bughio, H. R., Bughio, M. S. and Mastoi, N. N. 2002. Optimum Plant Density for High Yield in Rice (Oryza sativa L.). Asian Journal of Plant Sciences. Vol. 1 No. 1: 25-27.

Das, D.K., and Ram N 2006. The nature of humid substances under long-term manuring and fertilization in a rice 
wheat system. International Rice Research Notes; 31 (1): 29-31.

Das, P.K., Jena, M.K. and Sahoo, K.C. 2002. Effect of integrated application of vermicompost and chemical fertilizer on growth and yield of paddy in red soil of South Eastern Ghat Zone of Orrisa. Environment and Ecology, 20 (1): 1315.

Dey, R.C., Haloi, B., Ghose, T.J. and Chetia, S.K. 2006. Production of rice hybrid and yielding varieties under rainfed shallow ecology.Oryza 43(1): 51-54.

Dixit, K.G., and Gupta, B.R. 2000. Effect of farm yard manure, chemical and biofertilizers on yield and quality of rice (Oryza sativa) and soil properties. $J$. Indian Soc. Soil Sci., 48 (4): 773-780.

Dutta, M., and Chauhan, B.S. 2010. Effect of nutrient management practice on the performance of upland rice in a newly developed terraced land. Indian Agriculture; 54 (2): 13-21.

Dwivedi, A.P., Dixit, R.S. and Singh, G.R. 2006. Effect of nitrogen, phosphorus and potassium levels on growth, yield and quality of hybrid rice (Oryza sativa L.). Oryza, 43 (1): 64-66.

Fr. Henri de Laulamie, 1993. Developing the rice cultivation method SRI in Mada gascar: A developing Dialogue with farmers. Karthala 2003 pp 284. Retrieved 25 September 2016 pp.66. The fret documents Title $\mathrm{L}$ analysis de I elaboration du rendement duriz and was edited in January 1987 by a young French Agronomist, himself from the same institution as de Laulanie, promotion, 1983.

Gautam, O., Sharma, G.D., Rana, R. and Lal, B. 2012. Performance of hybrid rice INM in SRI under mid-hill condition of HP. Journal. Agriculture Sci. Carnbridg; 32(2): 52-53.

Islam, M.S., Sikdar, M.S.I., Rahman, M.M., Akhter, M.M. and Azad, A. K. 2008.
Performance of aromatic rice varieties as influenced by spacing. Journal of innovation and development strategy. 1(1): 43-46.

Kumar, M., Yaduvanshi, N. P. S. and Singh, Y. V. 2012. Effect of integrated nutrient management on rice yield, nutrient uptake and soil fertility status in reclaimed sodic soil. J. Indian Soc. Soil Sci., 60 (2): 132-137.

Limochi, K., 2012. Study of winter and summer planting dates on the flag leaf anatomy and yield of rice varieties in Khuzestan. M.Sc. Thesis of Agronomy, Collage of Agricultural, Islamic Azad University, Dezfoul, Iran. pp, 186.

Mirza B.B., Zia M.S., Szombathova N. Zaujec A. 2005. Rehabilitation of soils through environmental friendly technologies: role of sesbania and farm yard manure, Agriculture tropical and subtropical; 38 (1) 120-127.

Rath, P.C., and Marndi, B.C. 2010. Evaluation of resistance in some rice germplasm against white backed planthopper sogatella furcifera. Indian J. Plant Prot., 38 (2): 5-8.

Rathi, K.S., and Narain, J. 1990. Studieds on the effect of plot nsize and fretiliy levels on growth and yield of rice. M.Sc(Ag) Thesis, C.S.A.U.T. Kanpur.

Shankar, L., Laware 2011. Effect of organic fertilizer on growth and yield components in rice (Oryza sativa L.) Journal of agricultural science; 3 (3): 217-224.

Sharma, A.R., and Mitra, B.N. 1992. Integrated nitrogen management in rice (Oryza sativa) wheat (Triticum aestivum) cropping system. Indian J. Agril. Sci., 62 (1): 70-72.

Shekara, B.G., Shivakumara, G.B., Manjunath, B., Malikarjuna, N., Sudarashan, G.K. and Ravikumar, B. 2011. Effect of different level and time of nitrogen application on growth yield 
and nutrient uptake in aerobic rice (Oryza sativa L.). Environment and Ecology, 29 (2A): 892-895.

Singh, Braham and Singh, R.V. 2008. Comparative performance of rice hybrid sat different fertility levels under irrigated transplanted condition. International J. Agril. Sci., 4 (2): 485488.

Singh, S.R., and Verma, L.P. 2006. Effect of source and method of phosphorus application on growth and yield and protein content of transplanted rice (Oryza sativa L.). J. Environ. And Ecology, 24 (5): 315-319.

Stoop, W.A., and Kassam, A.H. 2005. The SRI controversy: A response. Field Crops Res., 91: 357-360.
Subhash Chander jitendra pandey Sharma, K.C., and Praveen Kumar 2005. Yield and quality of scented rice Pusa Banmati 1 (Oryza sativa L.) as influenced by nitrogen and herbicides under varying rice cultures. Annals of Agricultural Research; 26 (1): 24-34.

Tyeb, A., Paul, S.K. and Samad, M.A. 2013. Performance of variety and spacing on the yield and yield contributing characters of transplanted Aman rice. Journal of Agronomy for Environment. 7 (1): 57-60.

Vananja, M., and Raju, A. S. 2002. Integrated nutrient management-performance in rice crop. Annals of Agricultural Research; 23 (1):51-56.

\section{How to cite this article:}

Harikesh, Akhtar Ali, Shivam, Ravi Pratap Yadav, Sanjay Kumar, Ajit Kumar and Atul Yadav. 2017. Effect of Integrated Nutrient Management and Plant Geometry on Growth and Quality of Rice (Oryza sativa L.) Varieties under SRI Technique. Int.J.Curr.Microbiol.App.Sci. 6(10): 2503-2515. doi: https://doi.org/10.20546/ijcmas.2017.610.294 